\title{
LA COMPOSANTE LENTEMENT VARIABLE DES RAYONS $X$ SOLAIRES EN RELATION AVEC LA STRUCTURE DES CENTRES D'ACTIVITÉ
}

\author{
R. Michard et Mme E. Ribes* \\ (Observatoire Meudon, France)
}

\begin{abstract}
The slowly varying component of solar X-rays has been studied through records of the satellite Explorer-30 instrumented by the U.S. Naval Research Laboratory. Correlations with plage areas, and flux at radiofrequencies have been studied. High flux values in the 8-20 $\AA$ and, still more, the 1-8 $\AA$ bands are related to specific ARs, also characterized by great flare productivity, high intensities on $3 \mathrm{~cm}$, and anomalous magnetic structure.
\end{abstract}

Le rayonnement $\mathrm{X}$ du soleil peut être approximativement divisé en trois composantes:

(1) une composante stable due au rayonnement thermique de l'ensemble de la couronne,

(2) une composante lentement variable (CLV) due à l'émission des centres d'activité,

(3) les sursauts directement associés aux éruptions chromosphériques.

A l'aide des mesures effectuées par le satellite Solrad-8 $=$ Explorer-30 $=1965-93 \mathrm{~A}$ de l'USNRL, et reçues par les stations du Centre National d'Etudes Spatiales, nous avons étudié la CLV pour le semestre mars-août 1966. Dans certains cas les mesures du réseau du CNES ont été complétées par celles publiées dans Solar Geophysical Data.

Un indice journalier du flux solaire moyen dans les bandes $44-60 \AA, 8-20 \AA$ et 1-8 $\AA$ a été calculé en utilisant autant que possible exclusivement les observations effectuées en dehors des sursauts. La Figure 1 indique les variations de cet indice pour les deux bandes de plus courtes longueurs d'ondes. On constate la très grande variabilité du flux $\mathrm{X}$, bien que l'élimination des sursauts et le calcul des moyennes journalières réduisent considérablement les variations réelles.

La 'composante stable' est totalement indécelable sur 1-8 $\AA$ et presque négligeable dans la bande 8-20 ̊. Il n'en est pas de même sur 44-60 ̊ où un flux important reste présent même durant les minimums d'activité.

On notera aussi sur la Figure 1 d'importantes variations de la 'couleur' du rayonnement $\mathrm{X}$ solaire définie par le rapport des flux 1-8 et 8-20 $\AA$. Ce rapport est $<0 \cdot 01$

* Presented by P. Simon.

Kiepenheuer (ed.), Structure and Development of Solar Active Regions, 420-430. C I.A.U. 


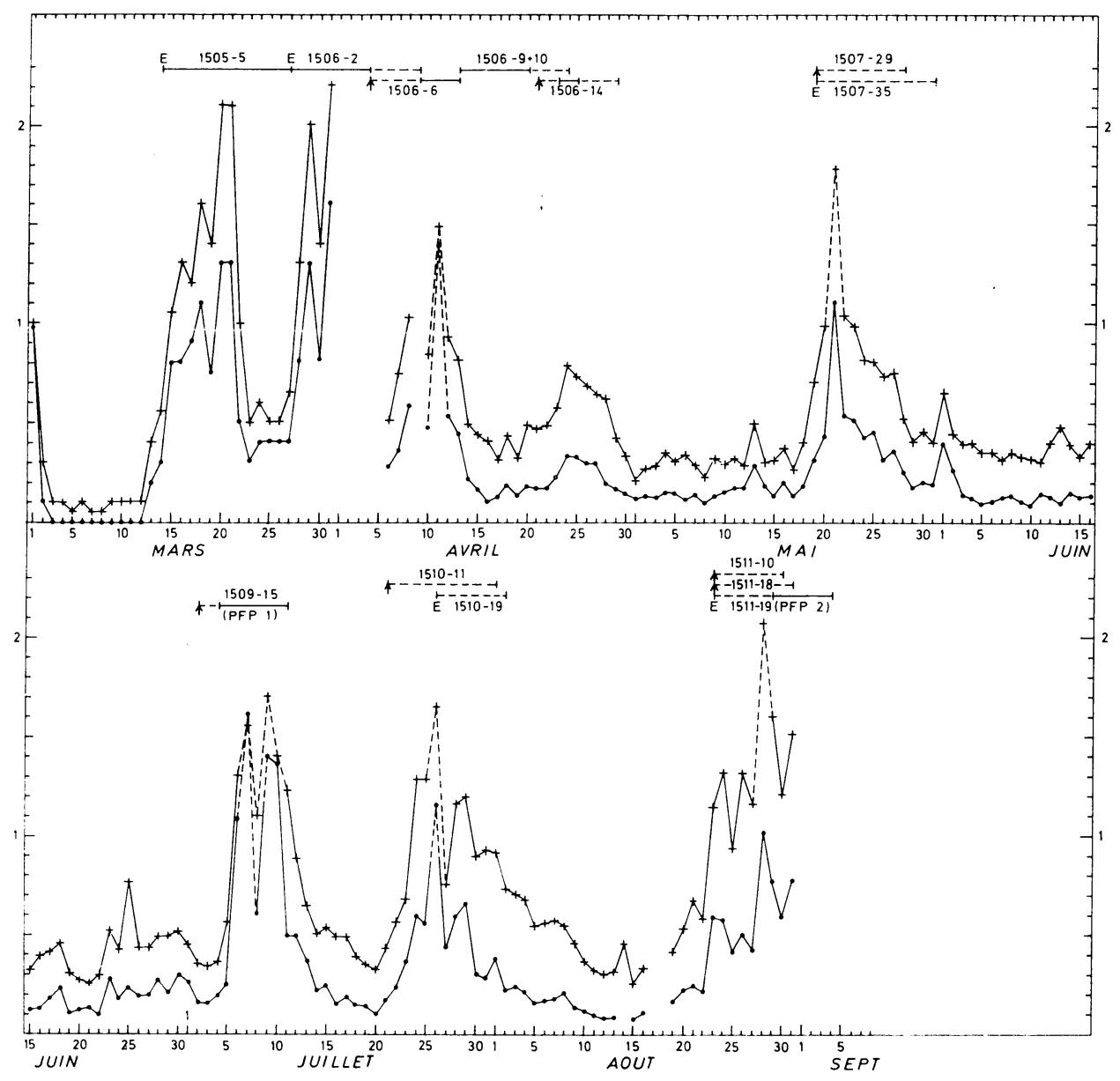

FIG. 1. Evolution de la composante lentement variable des flux X solaires de mars à septembre 1966 dans les bandes 8-20 et 1-8 A. Les points sont les moyennes journalières de mesures effectuées en dehors des sursauts associés aux éruptions, et recues par les stations du Centre National d'Etudes Spatiales. Les données de mars proviennent de l'USNRL (Solar Geophysical Data). En traits interrompus: mesures douteuses.

En haut sont indiqués les passages de CA (cités dans le texte et dans l'Appendice) responsables de l'émission au cours de diverses périodes remarquables. En trait plein: périodes où un seul CA produit tout le flux $X$ dans les bandes considérées. $E=C A$ passant au bord est. Flèche $=C A$ naissant sur le disque. 
pour des périodes très calmes (mars $3-13$ ); il peut atteindre des valeurs proches de $0 \cdot 1$ pour des périodes d'intense activité, tandis que sa valeur moyenne est de 0.042.

On peut également examiner la question de la 'couleur' du rayonnement $\mathrm{X}$ à l'aide des diagrammes de corrélation entre les flux de diverses bandes spectrales. Si la couleur était constante, c'est-à-dire si la température des sources coronales du rayonnement $X$ était invariable, la corrélation entre les flux 1-8 $\AA$ et 8-20 $\AA$ serait linéaire, avec une dispersion due seulement aux erreurs de mesure. La Figure 2 montre qu'il n'en est pas ainsi. La courbure de ce diagramme de corrélation indique que la température des zones coronales émissives croît généralement en même temps que leur

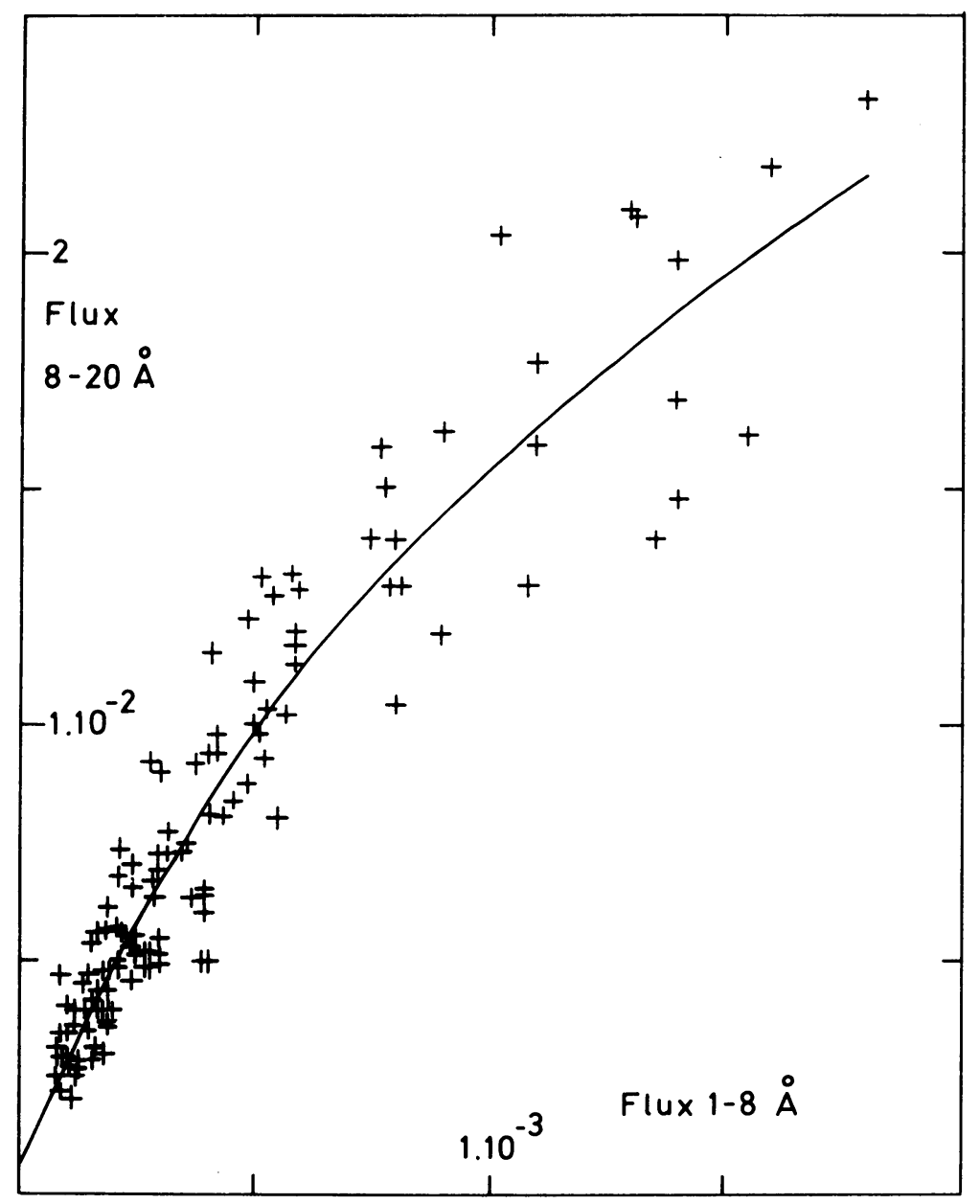

FIG. 2. Diagramme de corrélation entre fux $1-8$ et 8-20 A de la composante lentement variable. Les points sont les moyennes journalières de mars à août 1966. 


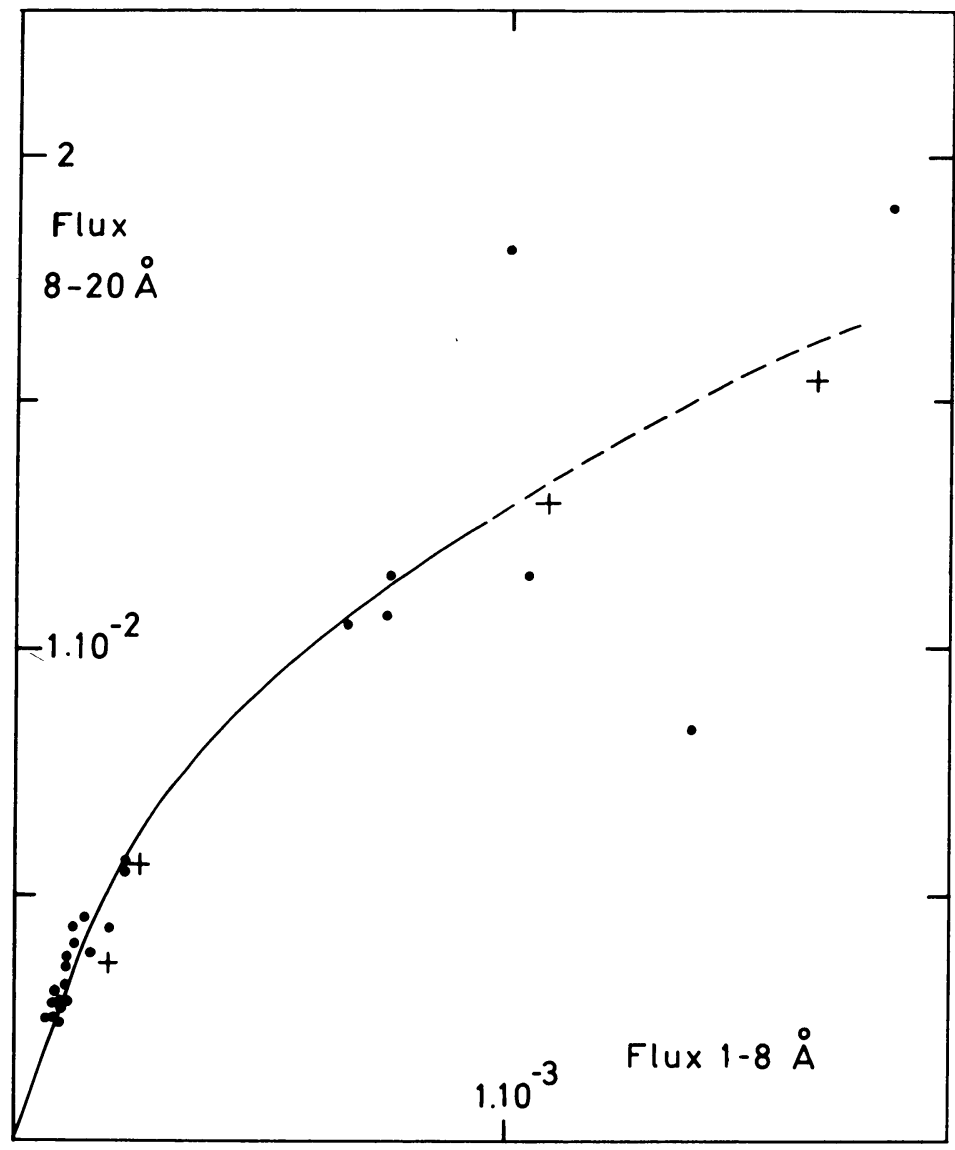

Fig. 3. Corrélation entre flux 1-8 et 8-20 A (hors sursauts) des 3 au 6 juillet 1966. Points $=$ mesures de l'USNRL (Friedman et Kreplin, 1967). Croix-Moyennes journalières de l'Observatoire de Meudon.

densité, le rayonnement $\mathrm{X}$ de la CLV devenant plus dur quand son intensité augmente. Le même phénomène est mis en évidence de manière encore plus sensible sur les diagrammes de corrélation portant sur des époques choisies: la Figure 3 est relative à la période du 3 au 6 juillet, phase de croissance du centre actif 1509-15 (McM 8362) qui produisit l'éruption à protons du 7 juillet (Friedman et Kreplin, 1967). Les diagrammes de corrélation entre flux $1-8$ et flux 44-60 $\AA$ indiquent également le net 'durcissement' des émissions solaires dues aux sources les plus intenses (Figure 4).

Les variations du flux X avec le cycle solaire (Kreplin et Gregory 1966), les photographies directes (Friedman, 1963; Underwood et Muney, 1967), l'étude des éclipses (Landini et al., 1966) indiquent que la CLV des rayons X provient des centres d'activité (CA). Mais il est évident que l'intensité et le spectre de cette émission sont prodigieusement variables d'un CA à un autre. Nous avons essayé de relier l'émission 


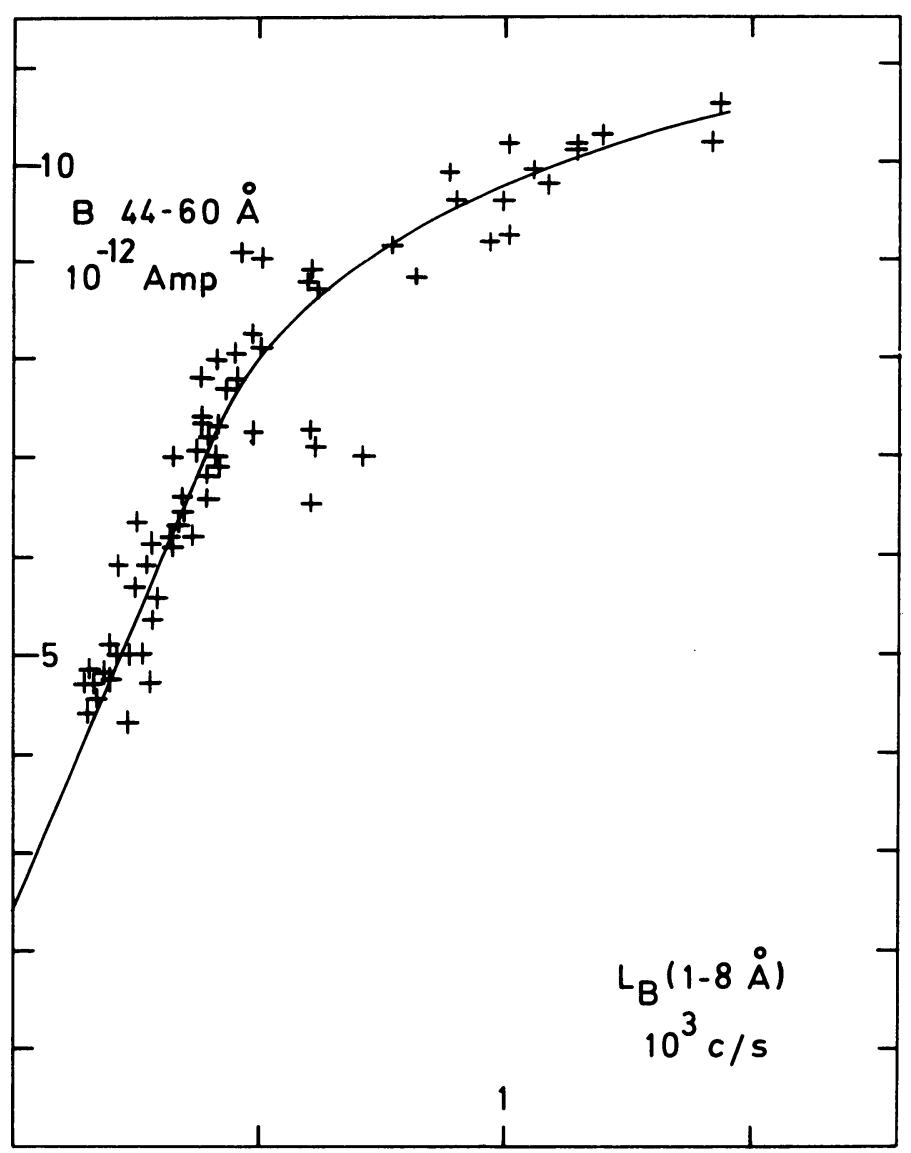

Fig. 4. Corrélation entre flux 1-8 et 44-60 A (hors sursauts) d'après les mesures du CNES pour août 1966.

$\mathrm{X}$ des $\mathrm{CA}$ à leurs caractères optiques et radioélectriques. Pour cela on peut faire appel à des techniques de corrélation, on peut aussi examiner directement les cas où un seul CA est responsable de la quasi-totalité du flux observé. Ces cas sont relativement fréquents durant la phase d'activité solaire modérée que nous étudions: les périodes correspondantes ont été notées sur la Figure 1.

La Figure 5 donne le diagramme de corrélation entre le flux 8-16 $\AA$ et l'aire des taches, corrigée de la perspective, d'après les mesures de l'Observatoire de RomeMonte Mario, corrélation que l'on peut qualifier d'assez bonne. Elle est en tout cas sensiblement meilleure que la corrélation entre le même flux et l'aire des plages du calcium pondérée par leur brillance. La Figure 6 nous donne un exemple d'une 'bonne' corrélation entre le flux X 8-16 $\AA$ et le flux radioélectrique à $3 \mathrm{~cm}$ mesuré par l'Observatoire de Toyokawa. 


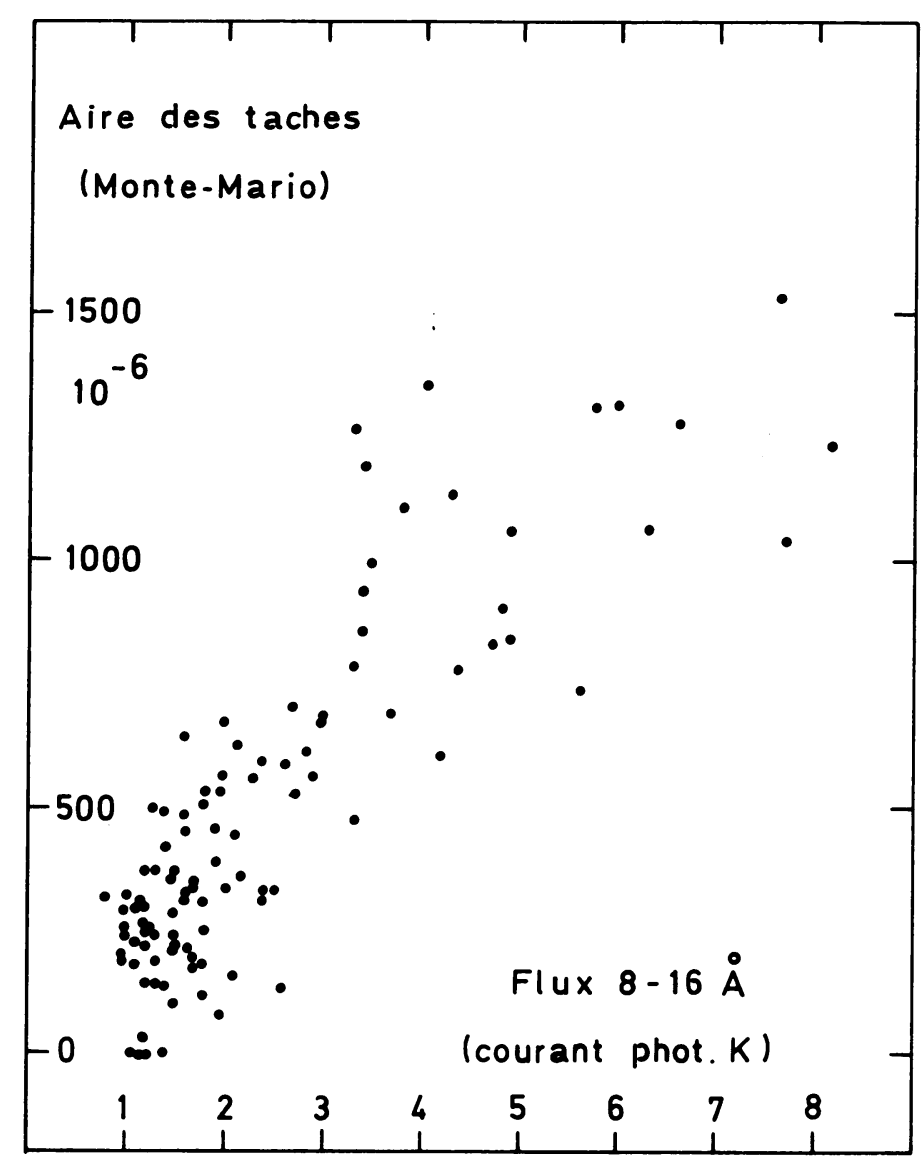

FIG. 5. Corrélation entre fux 8-20 A et aire des taches corrigée de la perspective (mesures de l'Observatoire de Rome).

Après ces exemples nous pourrons résumer les résultats de l'examen de nombreux diagrammes de corrélation dans le Tableau 1.

Les flux en rayons $X$ mous (44-60 $\AA$ ) sont très bien corrélés avec les flux radioélectriques aux fréquences voisines de $3000 \mathrm{MHz}$, assez bien avec l'aire des plages et médiocrement avec l'aire des taches. Quand on passe à la bande 8-16 $\AA$, la corrélation avec l'aire des taches s'améliore beaucoup mais celle liant le flux $\mathrm{X}$ à l'aire des plages se dégrade; d'autre part la corrélation avec le flux à $10000 \mathrm{MHz}$ reste excellente tandis qu'elle décroît beaucoup pour les fréquences plus basses. Pour les rayons $\mathrm{X}$ durs (1-8 $\AA$ ), toutes les corrélations deviennent beaucoup moins bonnes, leurs variations selon le paramètre considéré restant qualitativement semblables à celles du flux 8-16 .

L'étude de quelques cas particuliers permet d'expliquer le caractère assez vague des corrélations entre flux radioélectriques et rayons X durs. Les 10-12 avril 1966 par 


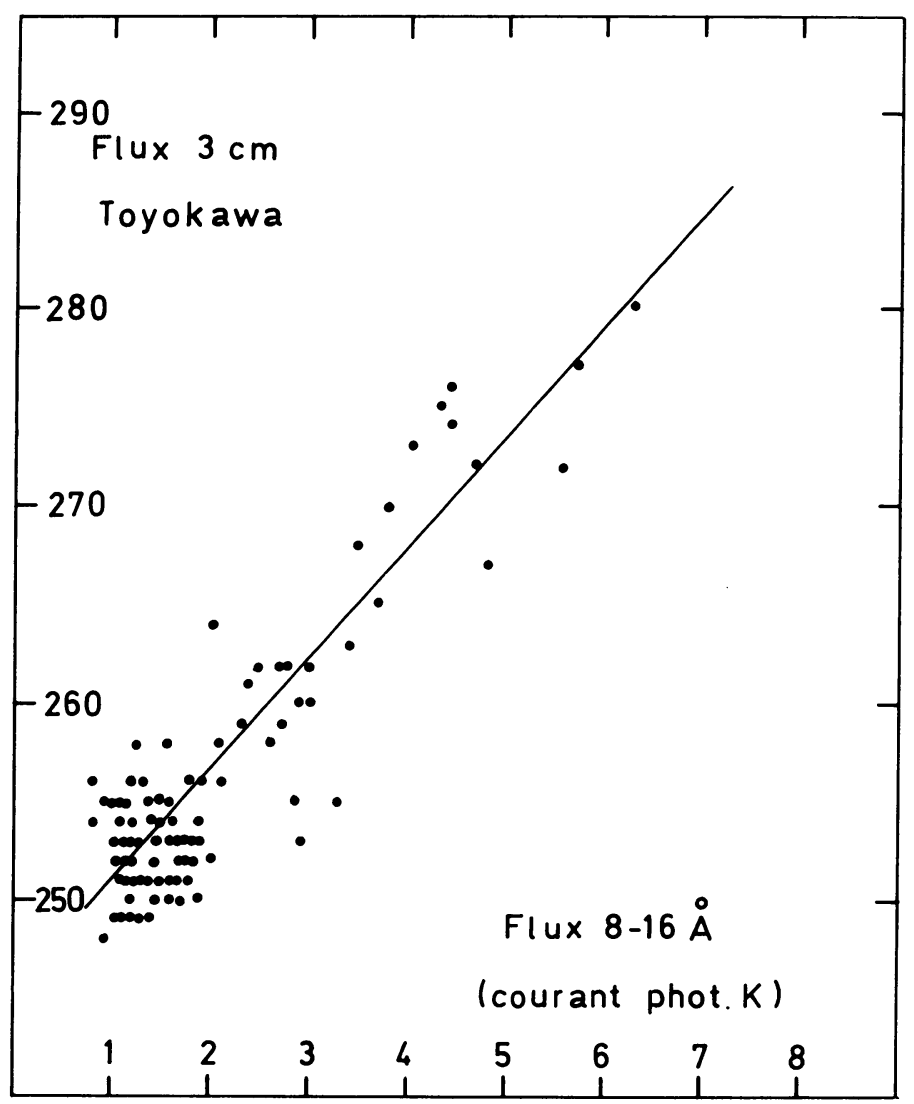

Fig. 6. Corrélation entre flux 8-20 A et flux radioélectrique sur $3 \mathrm{~cm}$ (mesures de l'Observatoire de Toyokawa).

\section{Tableau 1}

\section{Coefficients de corrélation entre flux $X$, indices solaires et flux radioélectriques}

Flux 1-8 Flux 8-16 Flux 44-60

Aire pondérée des plages Aire corrigée des taches (Rome)

Flux $3 \mathrm{~cm}$ (Toyokawa)

Flux $8 \mathrm{~cm}$ (Toyokawa)

Flux $10.7 \mathrm{~cm}$ (Ottawa)

Flux $14 \mathrm{~cm}$ (Toyokawa)

$\begin{array}{lll}0.28 & 0.57 & 0.77 \\ 0.59 & 0.81 & 0.70 \\ 0.60 & 0.87 & 0.82 \\ 0.58 & 0.84 & 0.88 \\ 0.49 & 0.77 & 0.88 \\ 0.40 & 0.66 & 0.85\end{array}$



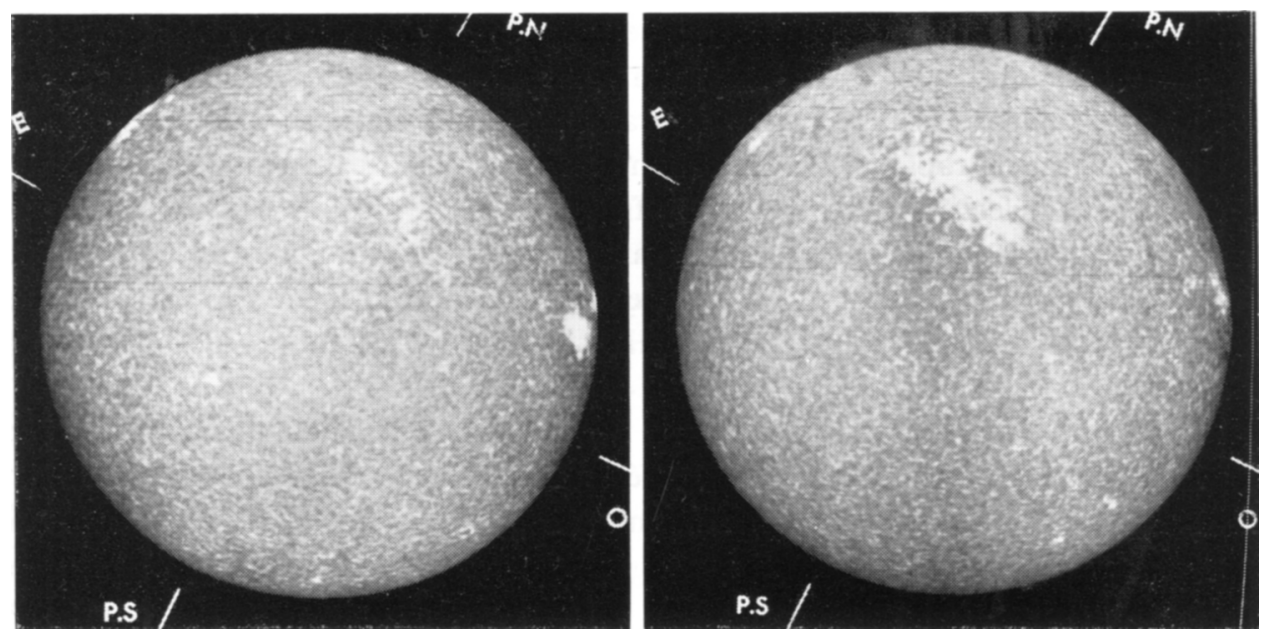

Fig. 7. Comparaison des CA visibles les 10 et 17 avril 1966 en liaison avec les variations des flux $X$ et centimétriques entre ces deux dates.

11 avril 1966:

$\begin{array}{ll}8-20 & 1.48 \times 10^{-2} \\ 1-8 & 1.40 \times 10^{-3} \\ 2.800 \mathrm{MHz} & 94 \\ 3.750 & 108 \\ 10.000 & 261\end{array}$

$\begin{array}{lcc} & 8-20 & 0.31 \times 10^{-2} \\ & 1-8 & 0.12 \times 10^{-3} \\ \text { Ottawa } & 2.800 \mathrm{MHz} & 95 \\ \text { Toyokawa } & 3.750 & 102 \\ \text { id. } & 10.000 & 253\end{array}$

Ottawa
Toyokawa
id.
17 avril 1966:

Ottawa
Toyokawa
id.

exemple (Figure 7) nous observons des flux $\mathrm{X}$ intenses dus à la présence du petit $\mathrm{CA}$ 1506-6. Les 15-17 avril nous sommes en présence du groupe de CA 1505-9-10: cette région comporte une plage très vaste mais des taches petites, avec une activité éruptive modérée. Le flux $X$ est alors réduit d'un facteur 3 dans la bande 8-16 $\AA$ et supérieur à 5 dans la bande $1-8 \AA$, tandis que les flux centimétriques sont très voisins dans les deux cas. De même lors du passage de la région 1509-15 (3-10 juillet) la croissance des flux X est beaucoup plus spectaculaire que celle observée sur 8 ou $10 \mathrm{~cm}$.

\section{Discussion}

Il est clair que l'émission X 8-16 $\AA$ et plus encore $1-8 \AA$ est concentrée en des 'points chauds' du CA, alors que la CLV centimétrique, pratiquement insensible à la température, est plus directement contrôlée par la surface du CA (telle qu'elle apparaît sur les spectrohéliogrammes de la raie $\mathrm{K}$ ).

Les émissions à 8-10 cm tendent à s'associer au mieux avec l'émission X 44-60 correspondant à des températures relativement basses. L'émission à $3 \mathrm{~cm}$ paraît plus sensible que celles de plus basse fréquence à des propriétés des CA autres que leurs 
surface (Avignon et al., 1966), telle que leur structure magnétique. Aussi ses corrélations avec les émissions $X$ à $8-16 \AA$ et même $1-8 \AA$ sont-elles meilleures. Pour des longueurs d'ondes radioélectriques plus grandes $(14$ et $30 \mathrm{~cm})$ les corrélations avec les flux $\mathrm{X}$ se dégradent rapidement. Il serait intéressant d'étendre ces comparaisons à des émissions radioélectriques de très haute fréquence. A ce propos notons que le $\mathrm{CA}$ 1506-6 que nous avons signalé comme ayant un rayonnement $X$ anormalement élevé par rapport à sa dimension et à son intensité centimétrique, a aussi présenté une intensité anormalement grande sur $17 \mathrm{GHz}$ d'après les observations de Tsuchiya et Nagane (1967).

Nous avons identifié et examiné les CA responsables des maximums remarquables $\mathrm{du}$ flux solaire en rayons $\mathrm{X}$ durant la période considérée (cf. Appendice). De cet examen on peut conclure que ces maximums sont dus à la présence d'au moins un CA ayant les caractéristiques suivantes:

(1) comporter un groupe de taches en phase de croissance et d'évolution rapide;

(2) appartenir à la classe $\mathrm{C}$ (complexe) de la classification magnétique des Régions Actives de l'Observatoire de Meudon (avec une préférence pour les sous-classes $C_{p}$ et $\mathrm{C} \gamma$ (Martres et al., 1966);

(3) appartenir (en général) aux types magnétiques $\beta \gamma, \gamma$ et $\delta$ de l'Observatoire du Mount Wilson;

(4) produire un grand nombre d'éruptions par jour;

(5) présenter une intensité remarquable sur $3 \mathrm{~cm}$ d'après les observations interférométriques de la Station de Nançay.

Les corrélations étroites qui existent entre ces divers caractères sont d'ailleurs bien connues.

Les observations spectroscopiques de l'émission $X$ du soleil dans les bandes 8-20 (Blake et al., 1965; Culhane et al., 1967) et 1-8 $\AA$ (Friedman, 1967) étudiées ici, montrent qu'elle est due à des condensations coronales de température sensiblement supérieure à la température moyenne de la couronne. Il est intéressant de noter que la formation de ces condensations exceptionnelles est conditionnée par la structure magnétique spéciale du CA: géométrie complexe, forts 'gradients' du champ longitudinal aux changements de polarité, variations rapides. Il est probable que les propriétés magnétiques du CA influent à la fois sur les possibilités de piégeage des ions coronaux, et sur l'efficacité du processus de chauffage.

\section{Remerciements}

Notre reconnaissance va à nos collègues de l'USNRL qui nous ont fourni généreusement les données nécessaires à la réception des mesures d'Explorer-30, et aux ingénieurs et techniciens du Centre National d'Etudes Spatiales qui ont assuré cette réception. 


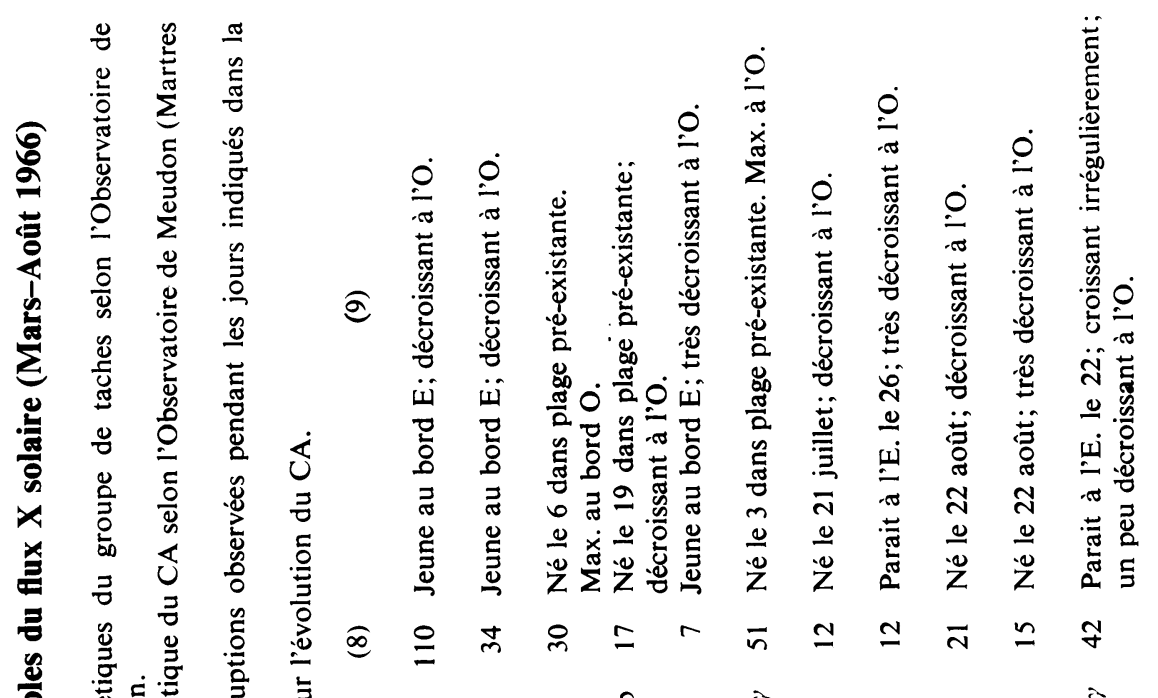

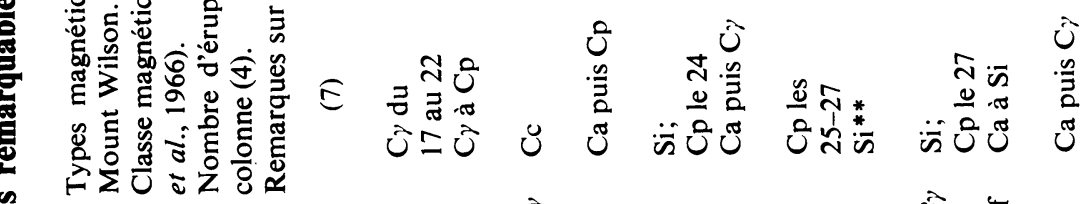

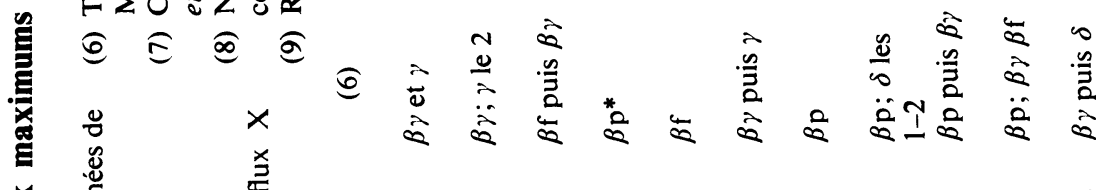

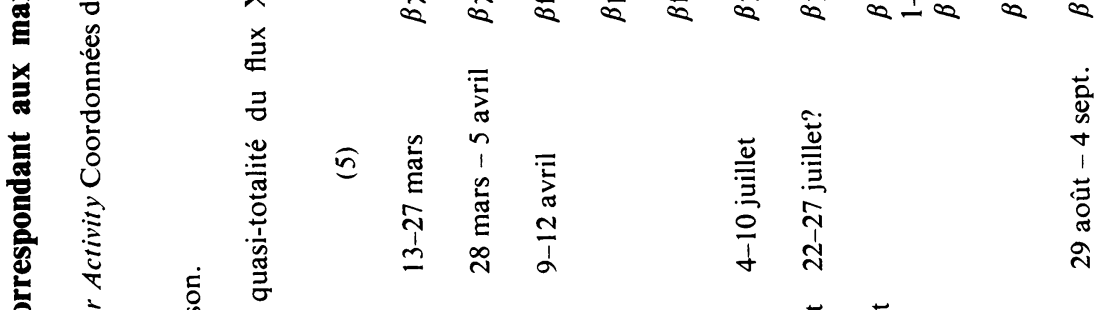

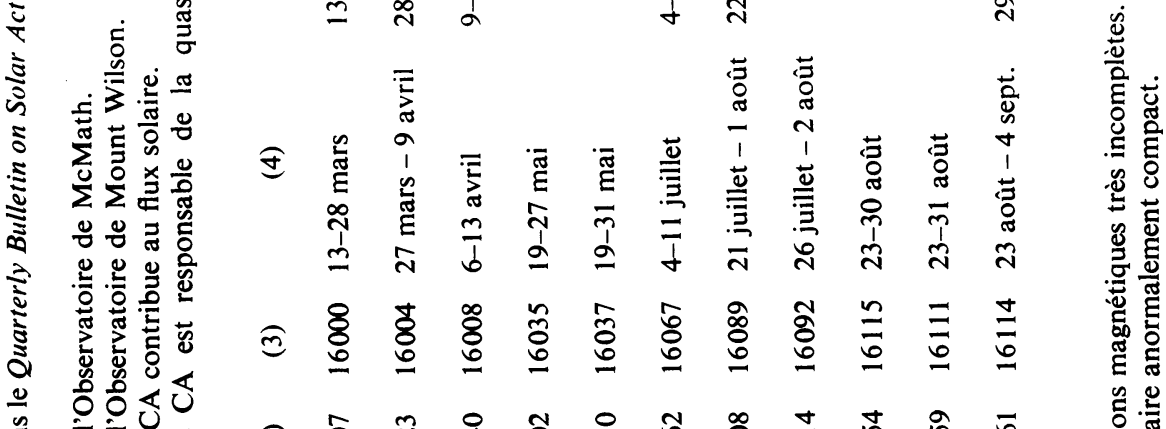

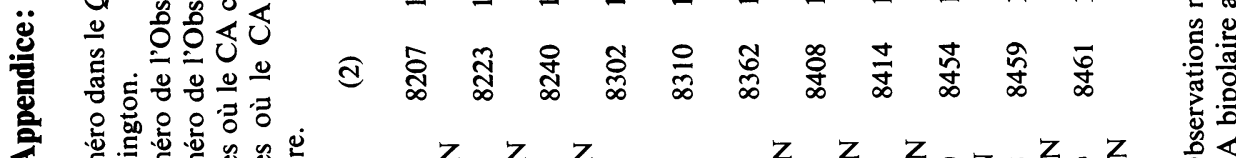

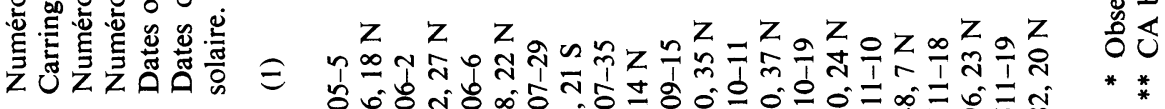

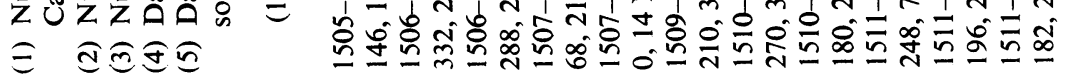




\section{Bibliographie}

Avignon, Y., Martres, M-J., Pick, M. (1966) Ann. Astrophys., 29, 33.

Blake, R. L., Chubb, T.A., Friedman, H., Unzicker, A. E. (1965) Astrophys. J., 142, 1.

Culhane, J.L., Evans, K., Pounds, K. A. (1967) Nature, 214, 41.

Friedman, H. (1963) Ann. Rev. Astr. Astrophys., 1, 59.

Friedman, H. (1967) communication personelle.

Friedman, H., Kreplin, R.W. (1967) 8th International Space Science Symposium, London.

Kreplin, R.W., Gregory, B. N. (1966) dans Space Research, VI, Spartan Books, Washington, p. 1011.

Landini, M., Russo, D., Tagliafferi, G. L. (1966) Nature, 211, 393.

Martres, M-J., Michard, R., Soru-Iscovici, I. (1966) Ann. Astrophys., 29, 245.

Tsuchiya, Asushi, Nagane, Kiyoshi (1967) Solar Phys., 1, 121.

Underwood, J.H., Muney, W.S. (1967) Solar Phys., 1, 129.

\section{DISCUSSION}

Neupert: Did you use sunspot number or area in your correlation with X-rays?

$P$. Simon: Sunspot area was used.

Krüger: How many values are used in your statistics of calculation of correlation coefficients?

P. Simon: One value each day.

Krüger: Do there exist some comparisons between the gradients of spectra of the s-component of solar radio emission and those of $\mathrm{X}$-ray emission?

P. Simon: Until now, there is no direct comparison, but it is obvious, according to the last part of this report, that there is a direct correlation between the X-ray spectrum and the centimetric spectrum.

Lundbak: Would you kindly explain how in the scheme presented the relation is established between the indices given and the $\mathrm{cm}$-values in the first column?

P. Simon: It is the conventional computation of the correlation function. 